\title{
STEP OUT WITH CONFIDENCE
}

\section{Alison Sewell}

Commonwealth Department of Health and Aged Care

The Commonwealth Department of Health and Aged Care commissioned a study of the information needs and perceptions of older Australians concerning falls injury and the prevention of falls. This article describes the findings of the study, which were used to inform the planning of communication activities for the National Falls Prevention for Older People Initiative, Step out with confidence.

\section{METHODS}

Fifty-nine older Australians from metropolitan and rural areas participated in the study. Some had experienced a fall, and some were the carers of those who had experienced a fall. Participants included individuals from culturally and linguistically diverse backgrounds. Information was obtained through seven group discussions and 10 in-depth interviews.

\section{RESULTS}

The investigation found that falls were generally known to be a problem among those aged 65 or older. However, there was considerable variation in the way that the problem was acknowledged by people in this age group. Typically, those more accepting of the personal risk of falling had already fallen or had experienced aged-related health problems that significantly affected their lifestyle. Having had a fall was found to be the most likely incident to trigger acceptance of personal risk: 'It has happened and it could happen again.' Older people who did not identify themselves as being 'old', and who had a more active lifestyle, tended to minimise their personal risk of falls and the relevance of falls prevention measures: 'It won't happen to me.'

\section{FACTORS PERCEIVED TO CONTRIBUTE TO FALLS}

The term 'fall prevention' was unfamiliar to the participants. However, the risk factors for falling that they identified included both extrinsic and intrinsic factors as follows:

\section{Extrinsic factors-or external to the individual:}

- tripping on steps, stairs, and uneven footpaths;
- slipping on polished floors, or on mats;

- poor footwear choices, such as high heels.

Intrinsic factors - or internal to the individual:

- hurrying and/or carelessness;

- feelings of being unsteady on their feet;

- poor vision.

Falls were attributed more readily to extrinsic factors, which are more tangible and more easily understood. Generally, older people felt that there is less they can do to prevent falls caused by intrinsic factors other than being very careful or using a walking aid.

\section{STRATEGIES TO PREVENT FALLS}

The extent and nature of falls prevention techniques adopted by participants was found to vary according to the perceived likelihood of falling. Many strategies were found to have been adopted for other reasons, with falls prevention being an additional benefit. Strategies adopted to prevent falls included:

- being more careful in the way that they do things;

- avoidance of certain situations that they perceived to be 'risky' in terms of falling;

- altering footwear to flat shoes and slip ons;

- increased use of walking aids.

While some individuals are prepared to use such aids, others are reluctant because they perceive the use of aids to signal acceptance of being old.

\section{CONCLUSIONS}

This research identified a clear need for easily accessible information for older Australians regarding falls prevention that:

- emphasises the preventable nature of falls;

- identifies the range of strategies that are available to prevent falls and how they prevent falls.

A full copy of the report: National falls prevention for older people initiative Step out with confidence: A study into the information needs and perceptions of older Australians concerning falls and their prevention is available from www.health.gov.au/ pubhlth/strateg/injury/falls/documents.htm. 